Research Article

\title{
An alternative approach to prepare alginate/acemannan 3D architectures
}

\author{
Simone S. Silva ${ }^{1,2}$ (1) $\cdot$ Luísa C. Rodrigues $^{1,2} \cdot$ Rui L. Reis $^{1,2,3}$
}

(c) Springer Nature Switzerland AG 2019

\begin{abstract}
Current research has explored biomedical applications using polymers and compounds derived from plant sources. In the present work, the interactions between acemannan, the main polysaccharide of aloe vera leaves, and alginate, a marine origin polymer were investigated to design biomaterials. As a proof-of-concept, alginate/acemannan beads were produced through an extrusion dipping method followed by freeze-drying technique to form porous beads. Characterization data confirmed that the $\mathrm{Ca}^{2+}$ presence in acemannan composition resulted in a stronger interaction between alginate and acemannan promoting gel formation and further the development of beads with a micro/nano roughness on their surface and heterogeneous porous formation in their interior. Results obtained until now suggest that the present approach represent a feasible and straightforward platform to design 3D biomaterial architectures for biomedical applications.
\end{abstract}

\section{Graphic abstract}

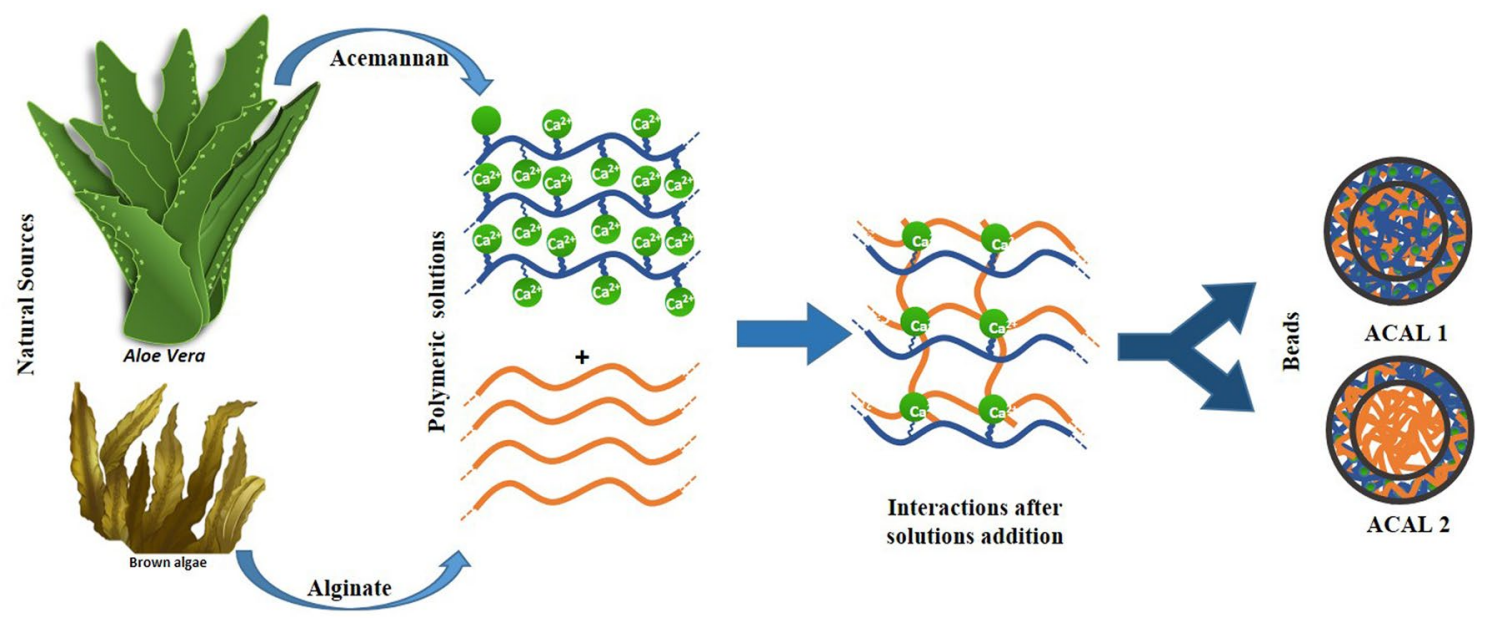

Keywords Acemannan · Alginate · Aloe vera

Simone S. Silva, simonesilva@i3bs.uminho.pt | 3 B's Research Group - Biomaterials, Biodegradables and Biomimetics, Univ. Minho, Headquarters of the European Institute of Excellence on Tissue Engineering and Regenerative Medicine, AvePark, 4805-017 Barco, Guimarães, Portugal. ${ }^{2}$ ICVS/3B's - PT Government Associate Laboratory, Braga, Guimarães, Portugal. ${ }^{3}$ The Discoveries Centre for Regenerative and Precision Medicine, Headquarters at University of Minho, Avepark, 4805-017 Barco, Guimarães, Portugal. 


\section{Introduction}

In the last years, there is a resurgent interest in the use of bioactive compounds derived from plants as alternative routes for biomaterials design. Aloe vera (AV), a medicinal plant, and its phytocompounds have provided reasonable means to design therapeutic strategies and biomaterials to treat diseases such as cancer, diabetes and skin repair [1, 2]. Previous studies demonstrated that the combination of plant extract, as AV gel, with natural polymers, e.g., chitosan (CHT) or alginate (ALG) resulted in matrices with improved properties [3, 4]. In this proposal, Acemannan (ACE) the main polysaccharide of AV leaves, which is formed by a long chain of acetylated mannose may be the principal responsible for the bioactivity achieved. Earlier works already have shown that ACE stimulate gingival fibroblast proliferation [5], cementoblast proliferation [6], and bone marrow stromal cell proliferation and differentiation in vitro [7]. These studies suggested that ACE can be an appealing material to create 3D structures for biomedical purposes. However, still, a low number of studies involving the production of ACE-based materials can be found in the literature $[8,9]$. The feasibility of ACE combination with $\mathrm{CHT}$ was tested to form mixed gels [8]; while the modification of ACE through methacrylation was proved and applied in the production of ACE sponges [9]. By its turn, the ALG, a marine polymer composed by $\beta(1 \rightarrow 4)$-linked D-mannuronic acid and $\mathrm{a}(1 \rightarrow 4)$-linked L-guluronic acid, possess high stability in physiological conditions [10]. Besides its controllable gelation in the presence of di- or polyvalent cations, alginate has desirable properties including biocompatibility, immunogenicity, and low toxicity [11]. ALG by itself can be processed as hydrogels, micro/nanoparticles, nanocomposites, and beads envisioning various applications such as wound healing, protein delivery, and cell encapsulation [11-13]. Moreover, ALG has been already combined with $\mathrm{CHT}$, for instance, to build polyelectrolyte complexes, which provided the production of materials such as films, hydrogels, and beads with enhanced stability in physiological conditions when compared to those based on a single polymer [14-16].

Thus, both ALG and ACE are naturally occurring polysaccharides, biodegradable, and biocompatible being these the main reasons for their choice. As a rational of this work, it was hypothesized that ALG gelification would occur when it is in contact with $A C E$, without the need of additional solvents, as commonly occur [17] since ACE possess $\mathrm{Ca}^{2+}$ traces in its composition, which will allow its interaction with ALG and, their further biomaterials processing. Beyond that, it is envisioned that the interactions established among those polymers can result in matrices with increased mechanical strength and stability when compared to each component. Similarly to the exploration of alginate-based materials for different biomedical applications namely cell encapsulation, drug delivery and tissue regeneration [17], we believe that this alternative approach could also be useful for designing biomaterials with improved properties. In this context, as a proof-of-concept, ALG/ACE porous beads were prepared through an extrusion dipping method using ALG solutions into a coagulation bath composed of an ACE solution. Subsequently, the developed beads were submitted to freeze-drying as an approach to design 3D biomaterials.

\section{Experimental}

\subsection{Materials}

Commercially available acemannan (ACE, BiAloe $\mathrm{Mw}$ average $200 \mathrm{kPa}$ ), powder form, obtained from aloe vera extracts was a gift from Lorand Laboratories (Texas, USA). Low viscosity sodium alginate from brown algae (ALG, $\sim 250 \mathrm{CP}$ ) was supplied by Sigma-Aldrich (USA). All other reagents and chemicals involved in the preparation and characterisation were of analytical grade.

\subsection{Preparation of alginate/acemannan-based materials}

Beads composed of ALG and ACE were prepared using the extrusion dipping method (Fig. 1). After preliminary studies, where polymeric aqueous solutions were prepared at concentrations in the range of $1-5 \%(\mathrm{w} / \mathrm{v})$, the polymeric concentrations of 4 and 5 wt $\%$ for ALG (ALG4\%, ALG5\% respectively) and ACE 5 wt\% (ACE5\%) were selected. The desired polymeric solutions were prepared in parallel. As the first step, ALG solutions were mixed with ACE solutions in ratio 1:1. The ALG/ACE (ACAL) beads were prepared by dipping the ALG solutions using a syringe with $1 \mathrm{~mm}$ diameter into ACE $5 \%$. The formed beads were kept in the ACE coagulation bath overnight to complete its incorporation. Further, the materials were washed several times with phosphate buffer solution (PBS) to ensure a total removal or the weakly attached ACE molecules. Then, the beads were frozen at $-80^{\circ} \mathrm{C}$ overnight and freeze-dried for $48 \mathrm{~h}$. The ALG/ACE (ACAL) beads were then stored at room temperature (RT) under vacuum. From now on the prepared samples will be designated by abbreviators where the beads resulting from the combination of ALG4\% and ACE5\% will be represented by ACAL1 and the ones of the combination of ALG5\% with ACE5\% will be designated by ACAL 2 . 


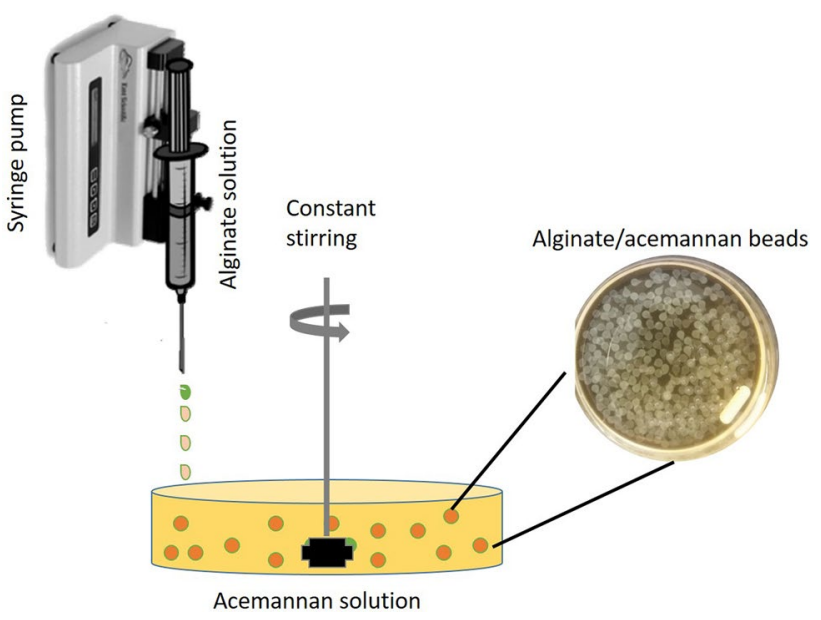

Fig. 1 Schematic representation of the formation of acemannanalginate beads

\section{Characterization}

\subsection{Rheology}

Rheological measurements were conducted on a Malvern Instruments Kinexus Rheometer equipped with a stainless steel parallel-plate sample holder of $25 \mathrm{~mm}$ in diameter and a $1 \mathrm{~mm}$ gap. To determine the linear viscoelastic region, the strain amplitude was measured in the shear rate range changing from 0.1 to $100 \mathrm{~Hz}$ in a $10 /$ decade rate, at a fixed temperature of $25^{\circ} \mathrm{C}$ and a constant $1 \%$ strain. The strain amplitude was optimized to ensure that measurements were performed in a linear viscoelastic region so that the elastic modulus ( $\left.G^{\prime}\right)$ and viscous modulus ( $\left.G^{\prime \prime}\right)$ were independent of the strain amplitude. For the measurement of strain dependency, $1.5 \mathrm{~mL}$ of blended solution was introduced into the sample holder, and $G^{\prime}$ and $G^{\prime \prime}$ was measured using a frequency sweep from 0.1 to $100 \mathrm{~Hz}$ at a constant temperature of $25^{\circ} \mathrm{C}$. The tests were done in triplicate.

Analysis of the frequency dependence of the phase angle, $\tan \delta$, were also made to provide more information about the rheological behavior of the systems. The phase angle can be determined using Eq. 1 [18]:

$\tan =G^{\prime \prime} / G^{\prime}$

\subsection{FTIR}

Fourier transform infrared (FTIR) spectra of the samples were obtained with a Nicolet 6700 Spectrometer. Samples were prepared as potassium bromide pellets at room temperature. The spectrum was collected by averaging 32 scans with a resolution of $4 \mathrm{~cm}^{-1}$, corresponding to the $4000-400 \mathrm{~cm}^{-1}$ spectrum region.

\subsection{X-ray spectroscopy (XPS)}

XPS samples analysis were performed on a Kratos AxisSupra instrument equipped with aluminium Ka monochromatized radiation at $1486.6 \mathrm{eV} \mathrm{X-ray} \mathrm{source,} \mathrm{within}$ ESCApe software. Due to the no conducting nature of the samples, it was necessary to use a co-axial electron neutralizer to minimize surface charging, which performed the neutralization by itself. Photoelectrons were collected from a take-off angle of $90^{\circ}$ relative to the sample surface. The measurement was done in a Constant Analyser Energy mode (CAE) with a $160 \mathrm{eV}$ pass energy for survey spectra and $40 \mathrm{eV}$ pass energy for high-resolution spectra. $\mathrm{C}$ 1s lower binding energy peak (285.0 eV) which corresponds to a hydrocarbon peak was used as a charge Ref. [19].

Data analysis and atomic quantification were determined from the XPS peak areas of High-resolution narrow scans using the ESCApe software supplied by the manufacturer Kratos Analytical. Double-sided carbon tape was used to fix samples to the sample holder.

\subsection{Scanning electron microscopy (SEM)}

The morphology of the alginate/acemannan beads was observed using a NanoSEM-FEI Nova 200 (FEG/SEM) scanning electron microscope. Before SEM analysis, samples were freeze-dried and further coated with gold using a Quorum/Polaron model E 6700 equipment and the analysis was performed with an acceleration voltage of $5.00 \mathrm{kV}$ and magnification from $150 \mathrm{X}$ to $4000 \mathrm{X}$.

\subsection{DSC}

The beads were subjected to differential scanning calorimetry (DSC) analysis under a flowing nitrogen atmosphere between 25 and $300{ }^{\circ} \mathrm{C}$ at a heating rate of $5{ }^{\circ} \mathrm{C} \mathrm{min}-1$ using a DSC Q100 from T. A. INSTRUMENTS instrument. Both temperature and enthalpy were calibrated with an indium standard at the same experimental conditions used for the studied materials. All samples, with a weight between 3 and $5 \mathrm{mg}$, were presented for DSC analysis in $40 \mu \mathrm{L}$ aluminum pans, which were covered with a suitable aluminum cover.

\subsection{Statistical analysis}

All quantitative experiments are run in triplicate, and results are expressed as a mean \pm standard deviation for $\mathrm{n}=3$. Two-way ANOVA statistical analysis was performed 


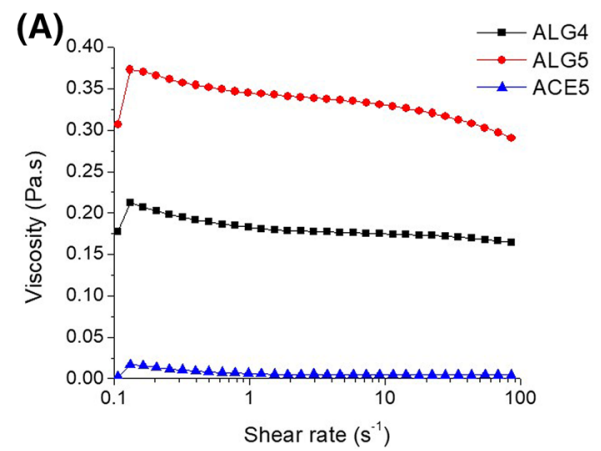

(D)

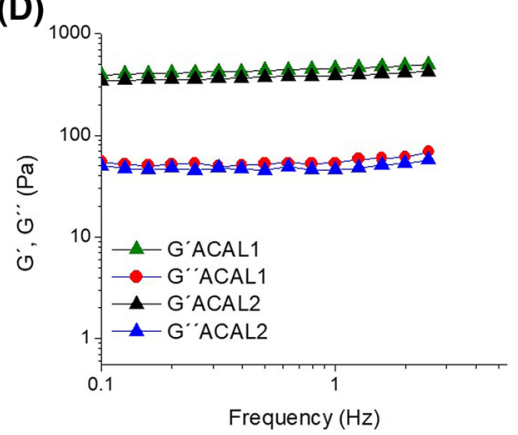

Fig. 2 Flow curves of the ALG4\%, ALG5\%, and ACE5\% solutions (A) and ACAL 1 and ACAL2 (B) produced at different shear rates. C Frequency sweep test for ACAL1 and ACAL2, a measurement made with the solutions just prepared; $\mathbf{D}$ frequency sweep test for ACAL1

with Bonferroni's post-test by using GraphPadPrism program (GraphPad Software, San Diego, http://www.graph pad.com). Differences with $p<0.05$ between the groups were considered to be statistically significant.

\section{Results and discussion}

In our approach, the combination of ALG, a gelling polymer, with ACE, a non-gelling one, was evaluated as an alternative pathway to prepare biomaterials. Sodium ALG is a water-soluble polymer which gives origin to an anionic solution, where its viscosity can be increased leading to a gel network formation in the presence of divalent ions such as $\mathrm{Ca}^{2+}[20,21]$. By its turn, ACE is neutral in aqueous solution, and it did not show evidence of gelling by itself. This polymer possesses $\mathrm{Ca}^{2+}$ traces in its composition as a residue of its extraction from AV leaves. Therefore, we explored the gel formation ability between ACE and ALG due to mixed junction zones between them, as commonly evidenced in ALG/CHT based systems [10, 16, 20, 22].

The viscosity of the solutions of ALG4\%, ALG5\%, ACE5\%, and the blended solutions (ACAL 1 and ACAL2) was evaluated as a function of the applied shear rate (Fig. 2A). As expected, the ACE solution presented lower viscosity when compared with ALG solutions, where differences

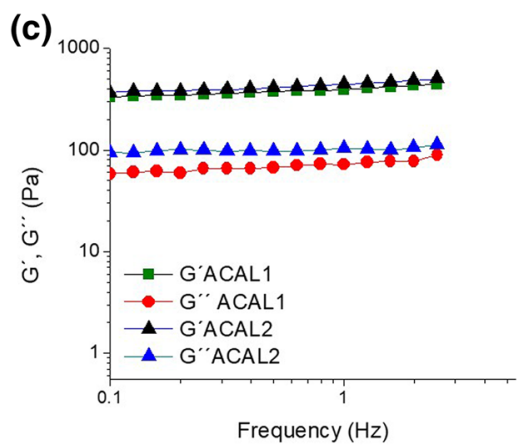

(E)

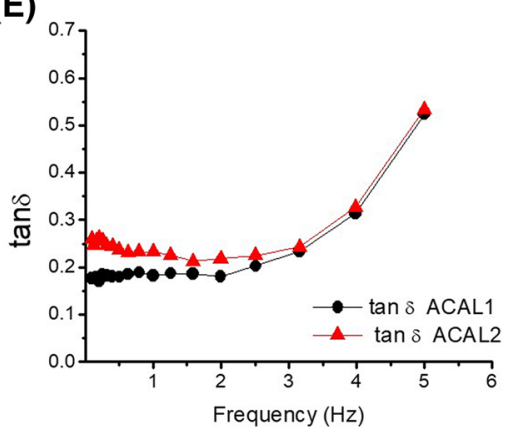

and ACAL2, a measurement made with the blended solutions after preparation ( $24 \mathrm{~h}$ ); and E loss tangents of the ACAL 1 and ACAL2 as a function of frequency

Table 1 Storage modulus $\left(\mathrm{G}^{\prime}\right)$ and loss modulus $\left(\mathrm{G}^{\prime \prime}\right)$ at $1 \mathrm{~Hz}$ and $25^{\circ} \mathrm{C}$

\begin{tabular}{llllll}
\hline System & \multicolumn{2}{l}{ Just prepared } & & \multicolumn{2}{c}{ After production $(24 \mathrm{~h})$} \\
\cline { 2 - 3 } \cline { 6 - 6 } & $\mathrm{G}^{\prime}(\mathrm{Pa})$ & $\mathrm{G}^{\prime \prime}(\mathrm{Pa})$ & & $\mathrm{G}^{\prime}(\mathrm{Pa})$ & $\mathrm{G}^{\prime \prime}(\mathrm{Pa})$ \\
\hline ACAL1 & $393 \pm 0$ & $72.0 \pm 0$ & & $491.3 \pm 50.9$ & $58.1 \pm 6.8$ \\
ACAL2 & $445 \pm 0$ & $103.6 \pm 0$ & & $354.8 \pm 45.5$ & $46.1 \pm 0.3$ \\
\hline
\end{tabular}

in viscosity are directly correlated to an increase of the polymer concentration. Both blended solutions displayed shear thinning behavior, in which the viscosity decreased with increasing shear rate (Fig. 2B). This behavior was expected as the shearing of the polymer chains disrupts the three-dimensional structure through the breaking of primary and secondary bonds.

Polysaccharide solutions are viscoelastic materials that exhibit solid and liquid characteristics, where storage $\left(G^{\prime}\right)$ and loss $\left(G^{\prime \prime}\right)$ moduli refer to the elastic and viscous response of a given material, respectively [23]. The results of dynamic frequency sweep tests for ACAL systems are presented in Fig. 2C-E and Table 1.

In frequency sweep tests, $G^{\prime}$ and $G^{\prime \prime}$ increased with the frequency in both systems, which is attributed to the interactions between the polymeric chains. Table 1 showed that both ACAL solutions have gel character, 
(A)

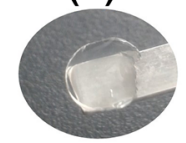

(B)

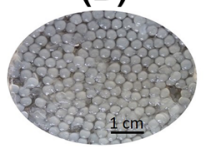

(C)
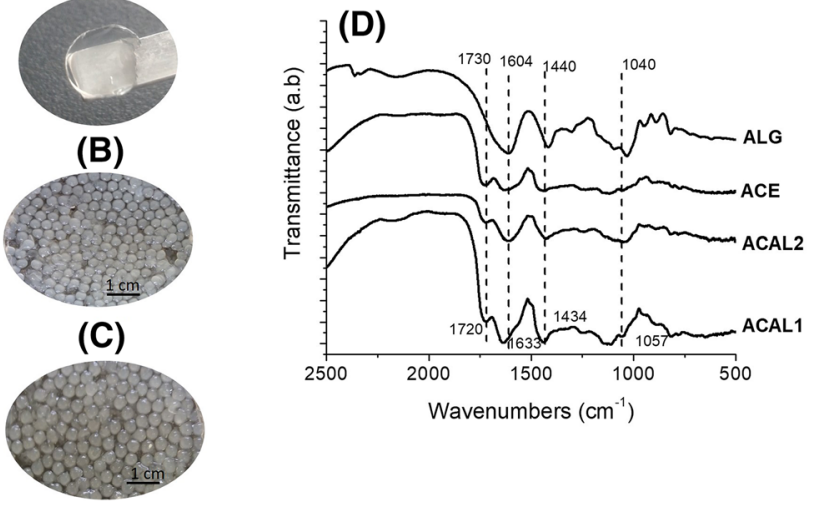

Fig. 3 Photographs of the alginate/acemannan based matrices: $\mathbf{A}$ ALG/ACE gel, B ACAL 1 beads, and C ACAL 2 beads; D FTIR spectra of the alginate, acemannan, ACAL 1 and ACAL2

demonstrated by $\mathrm{G}^{\prime}>\mathrm{G}^{\prime \prime}$, which indicated that the elastic behavior dominates over the viscous one. Furthermore, differences were observed in the rheological behavior in both systems $24 \mathrm{~h}$ after production (Table 1, Fig. 2D). In ACAL 1 , an increase of $G^{\prime}$ values was observed $24 \mathrm{~h}$ after the preparation, which indicated that the elastic properties of this gel became stronger along the time. Contrarily, a reduction of the $\mathrm{G}^{\prime}$ values was noted in ACAL 2. It is possible that the use of ALG 4\% in ACAL 1 promoted an increase of junction zones by $\mathrm{Ca}^{2+}$ ions (ACE), which led to a higher crosslinking density. Therefore, the use of ALG in different concentrations in the systems could be a useful tool to modulate the crosslinking density of junctions that govern the connectivity in the gel network.

An increase of the $\tan \delta$ as the frequency increase was observed for both ACAL systems (Fig. 2E), confirming the viscoelastic solid character of the system. Similar rheological behavior was observed for the CHT-ALG system [18]. The overall rheology response of the ACAL systems confirmed that the presence of ACE leads to a reinforcement of the mechanical properties of the gels, contrarily to results obtained in other systems containing $A C E$, as per example chitosan-ACE mixed systems [8].

\subsection{Beads formation and structural characterization}

A gel character of the ACAL solutions was evidenced by rheological analysis, as described above. Following up, a proper gel formation was investigated by mixing ALG solutions at different concentrations, namely 4 and $5 \%$, with an ACE solution at $5 \%$. The gel formation was obtained, as shown in Fig. 3A. Thus, the gel formation associated with the rheological behavior of the blended solutions suggests the existence of good interaction between the components of the systems.
ACAL beads (Fig. 3B, C) were obtained by dropping an aqueous solution of sodium ALG at $4 \%$ and $5 \%(\mathrm{w} / \mathrm{v})$ into the coagulation bath containing ACE $5 \%(\mathrm{w} / \mathrm{v})$. ACAL beads with a spherical shape and homogeneous size distribution were produced after ALG solution contact with the coagulation bath (ACE).

\subsection{FTIR}

The chemical structures of the samples were examined by FTIR spectra (Fig. 3D). The spectrum of ALG (Fig. 4) showed important absorption bands at 1605 and $1440 \mathrm{~cm}^{-1}$, which are linked to the asymmetric and symmetric stretching vibrations of carboxylate anions, respectively [24]. A representative FTIR profile of ACE (Fig. 3D) showed the presence of $C=O$ stretching vibration of the acetyl group at $1720 \mathrm{~cm}^{-1}$, COO- (corresponds to $1435 \mathrm{~cm}^{-1}$ ) and -COC (corresponds to $1124 \mathrm{~cm}^{-1}$ ) as the predominant functional groups [25]. In FTIR spectra of both ACAL beads, we observed the characteristic bands of ALG and ACE, proportionally to the concentration of the components. Small variations in the wavenumbers were also observed for the different signals of the ACAL beads that could indicate the existence of specific interactions between the components.

\subsection{XPS}

The chemical composition of the samples was examined by XPS surface measurements (Figs. 4, 5). From the highresolution spectra present in Fig. $4 \mathrm{ii}$, is possible to note that there were no apparent changes in the $C 1 \mathrm{~s}$ and $O$ $1 \mathrm{~s}$ region spectra of the samples ACAL1 and ACAL2 when compared with each other. Besides the similar quantification of those elements (Table 2), it was noticed that the chemical interactions established between the elements are also comparable, with no significant shift detected in the binding energies of the respective bands. The elements quantification achieved through XPS data analysis of ACE powder confirmed that ACE has about $1.22 \%$ of $\mathrm{Ca}^{2+}$ ions in its composition (Table 2 and Fig. 4i), similarly beside residual presence of sodium $(\mathrm{Na})$ in ACE powder sample, is evident the presence of this ion in the ALG powder analyzed representing 5.55\%. From this, the differential studied elements in these samples are $\mathrm{Na}(\mathrm{Na} 1 \mathrm{~s})$ and $\mathrm{Ca}$ (Ca 2p) (Fig. 5) which will allow prospecting the ACE distribution into the structure of the beads.

Regarding the quantification of chemical elements that compose the beads is suggested that the presence of the ACE in the ACAL2 formulation is mainly evidenced on the surface; while in ACAL1, its presence was observed in the inner of the beads (Tables 2,3). Nevertheless, ACE was found mainly as a coating onto the ACAL1 beads; it 
(i)
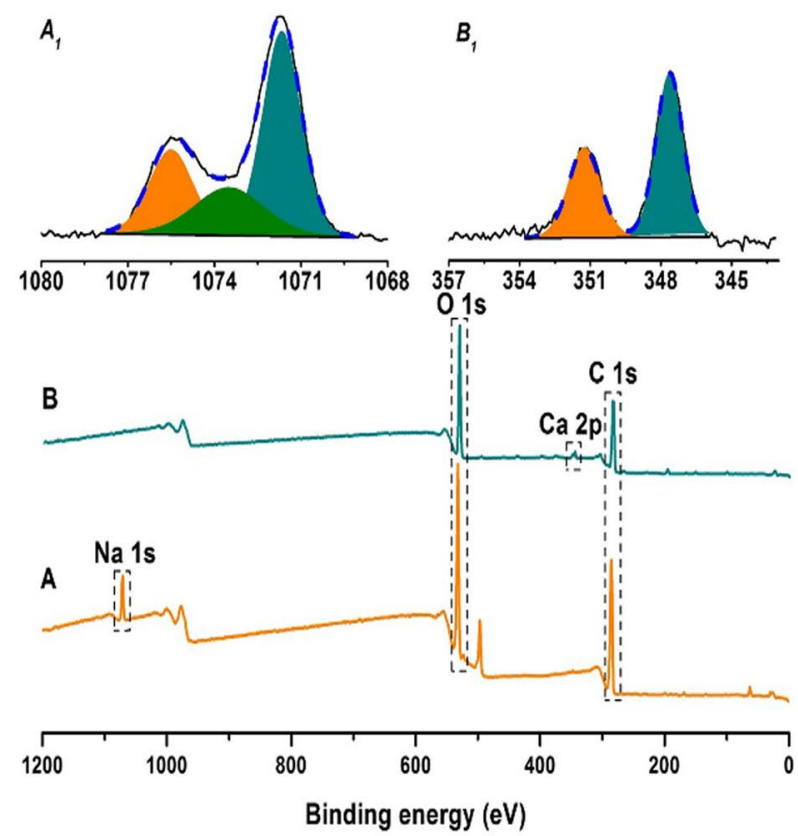
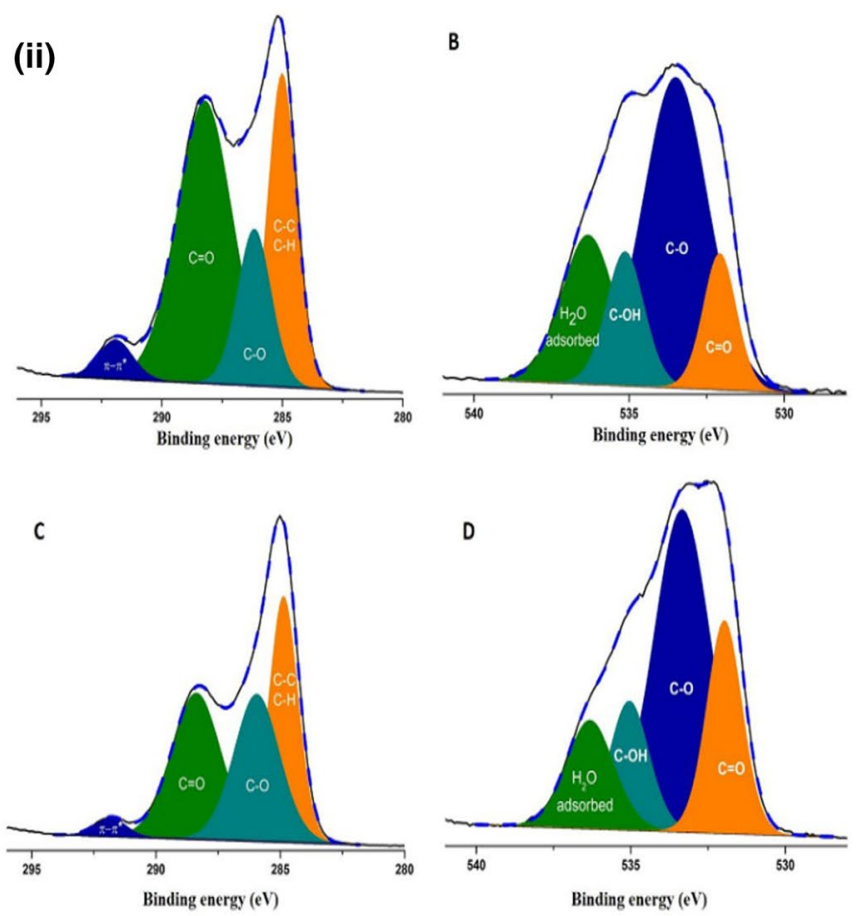

Fig. 4 i XPS wide scan of the polymers $A-A L G$ and B-ACE; inset: A1-Sodium region scan in ALG sample; B1-Calcium region scan of the ACE sample. ii XPS spectra of ACAL1 (A) C 1s, (B) O 1s; and ACAL2 (C) C 1s, (D) O 1s



Fig. 5 i XPS wide scan of the outer side of the prepared microparticles A-ACAL2 and B-ACAL1; inset: sodium region scan in ACAL2 (A1) and ACAL1 (B1) sample; and calcium region scan of ACAL2 (ii)

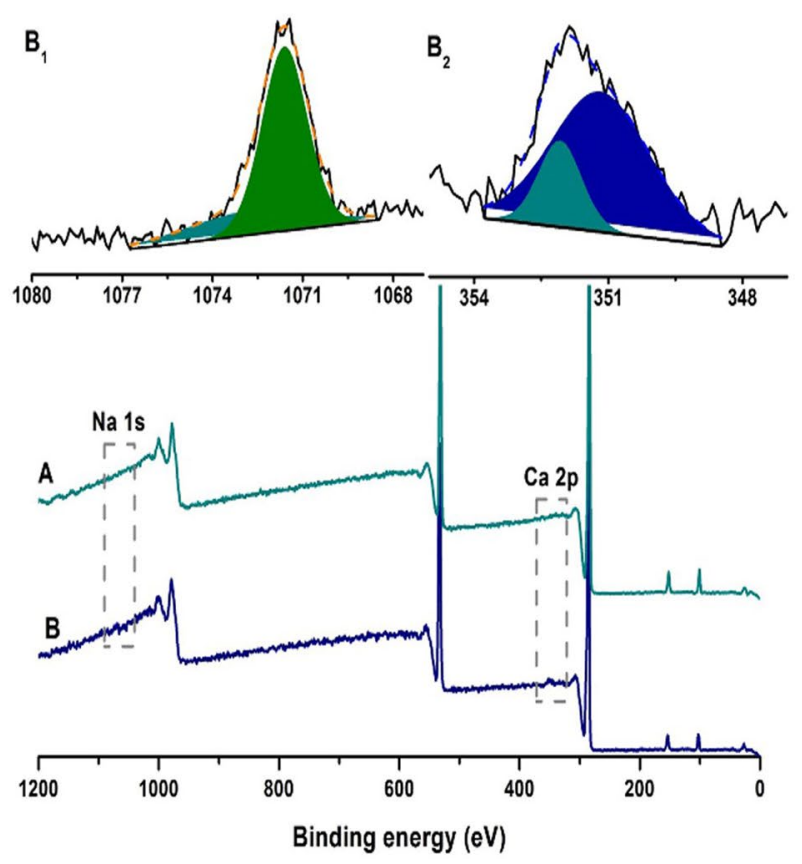

(A2) and ACAL1 (B2) sample. ii XPS wide scan of the inner side of the prepared beads $A-A C A L 2$ and $B-A C A L 1$; inset: $A C A L 1$ region scan of sodium (B1); and calcium (B2) 
Table 2 Quantification of chemical elements

\begin{tabular}{lllll}
\hline Sample/atomic percent & $\% \mathrm{C}$ & $\% \mathrm{O}$ & $\% \mathrm{Na}$ & $\% \mathrm{Ca}$ \\
\hline ACE & $62.71 \pm 0.10$ & $35.80 \pm 0.10$ & $0.27 \pm 0.03$ & $1.22 \pm 0.03$ \\
ALG & $59.81 \pm 0.12$ & $34.44 \pm 0.11$ & $5.55 \pm 0.08$ & $0.20 \pm 0.01$ \\
ACAL1 & $59.99 \pm 0.14$ & $37.38 \pm 0.13$ & $0.64 \pm 0.04$ & $2.00 \pm 0.04$ \\
ACAL2 & $59.03 \pm 0.21$ & $38.36 \pm 0.20$ & $1.02 \pm 0.08$ & $1.59 \pm 0.05$ \\
ACAL1 cross section & $76.91 \pm 0.47$ & $22.71 \pm 0.27$ & $0.21 \pm 0.03$ & $0.18 \pm 0.02$ \\
ACAL2 cross section & $78.59 \pm 0.11$ & $21.29 \pm 0.11$ & $0.09 \pm 0.04$ & $0.03 \pm 0.01$ \\
\hline
\end{tabular}

Table 3 Chemical bounds established with carbon

\begin{tabular}{lrrr}
\hline Sample/atomic percent & $\%(\mathrm{C}-\mathrm{C}, \mathrm{C}-\mathrm{H})$ & \multicolumn{1}{l}{$\%(\mathrm{C}-\mathrm{O})$} & \multicolumn{1}{l}{$\%(\mathrm{C}=\mathrm{O})$} \\
\hline ACE & $16.53 \pm 0.12$ & $29.76 \pm 0.15$ & $12.51 \pm 0.14$ \\
ALG & $12.81 \pm 0.12$ & $14.76 \pm 0.14$ & $16.84 \pm 0.18$ \\
ACAL 1 beads & $27.10 \pm 0.31$ & $8.32 \pm 0.53$ & $8.44 \pm 0.17$ \\
ACAL2 beads & $14.19 \pm 0.22$ & $27.71 \pm 0.27$ & $15.57 \pm 0.27$ \\
ACAL1 cross section & $9.10 \pm 0.29$ & $15.15 \pm 0.38$ & $37.37 \pm 0.47$ \\
ACAL2 cross section & $47.75 \pm 0.36$ & $2.75 \pm 0.14$ & $19.53 \pm 0.24$ \\
\hline
\end{tabular}

also has penetrated in the inner core of the beads, however in a lower proportion. These observed differences in ACE incorporation could be related to the differences of viscosity observed for ALG $4 \%$ and $5 \%$ solutions used in the production of ACAL 1 and ACAL2 beads, respectively. In the ACAL 2 beads formation, the high viscosity of the solution may prevent the penetration of the ACE to the inner part of the beads.

From the conclusions obtained by the XPS analysis about the reorganization of the ACE and ALG into the beads, it was possible to propose a schematic representation of beads structure that will be useful in the interpretation of other relevant features observed. It is assumed from previous studies involving AV [26], and from ACE composition analysis assays that the carboxylate moieties on ALG chains will interact with $\mathrm{Ca}^{2+}$ ions present in ACE promoting ALG gelation to form a $3 \mathrm{D}$ architecture. Moreover, the molecules of $\mathrm{Ca}^{2+}$ could be aggregated into the formed beads due to the interactions with alginate (egg-box formation), which in turn result in a higher percentage of detected $\mathrm{Ca}^{2+}$ ions, when compared to powder. Beside the egg-box established due to $\mathrm{Ca}^{2+}$, the combination of ALG and ACE chains also reveals in a lower degree of influence, the existence of mixing zones which result from a balance between hydrophilic (hydroxyl groups) and hydrophobic (carbonyl groups) interactions. Also, governing the association junctions into the networks is the evaporation step that guarantees a polymer concentration above the critical concentration for chain entanglement.

Figure $6 \mathrm{~A}$ shows the representation of the structure formed by ALG in association with ACE in ACAL1 and ACAL2.
XPS was also used to visualize the surface and crosssection of the samples (Fig. 6B). This analysis was useful to understand the distribution of chemistries across the sample surface. It seems that ACE has a more homogeneous distribution in both surfaces and cross-sections of ACAL1 than ACAL2.

\subsection{Morphology features}

SEM micrographs of both ACAL microspheres formulations are reported in Fig. 7A. The structures showed a micro/ nano roughness on their surface, and an open pore structure in the cross-sections. It seems that the size of the pores in ACAL2 is smaller than ACAL1, suggesting that the composition plays an effect on the morphology of the beads. The formation of stronger interactions between the components due to the presence of alginate in a higher percentage in ACAL2 as well as the increased viscosity of the $5 \%$ ALG solution could be the reasons for that. These features could represent a benefit for cell growth and tissue healing.

\subsection{Thermal properties}

The DSC thermograms of the ACE, ALG, and ACAL beads are shown in Fig. 7B, C. Under $\mathrm{N}_{2}$, all samples decomposed in three overlapping peaks. The first endothermic peak around $80^{\circ} \mathrm{C}$ suggests a dehydration process. ACE showed a sharp endothermic curve, with a peak of $164.3^{\circ} \mathrm{C}$, followed by another endothermic peak at ca $303^{\circ} \mathrm{C}$. This sequence is attributed to polymer decomposition.

Moreover, ALG showed an exothermic peak at ca $241.9^{\circ} \mathrm{C}$ that was ascribed to pyrolysis of the sample. The beads have one endothermic peak at $179.7^{\circ} \mathrm{C}$ and one exothermic peak at $277.3^{\circ} \mathrm{C}$ (ACAL1); and one endothermic peak at $182.7^{\circ} \mathrm{C}$ and one exothermic peak at $273.1^{\circ} \mathrm{C}$ for ACAL2. The exothermic peaks of the ACAL beads were higher than the peak value of the ALG and ACE, which suggested a stronger interaction between the polymers, which resulted in an increase of their resistance to the thermal degradation. 
(A)

$$
\text { }
$$

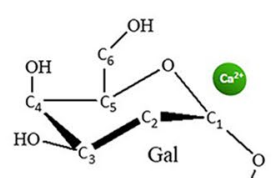

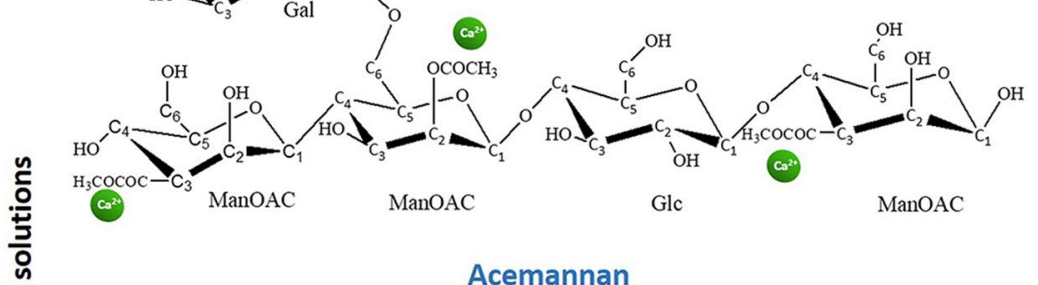

$\frac{5}{2}$
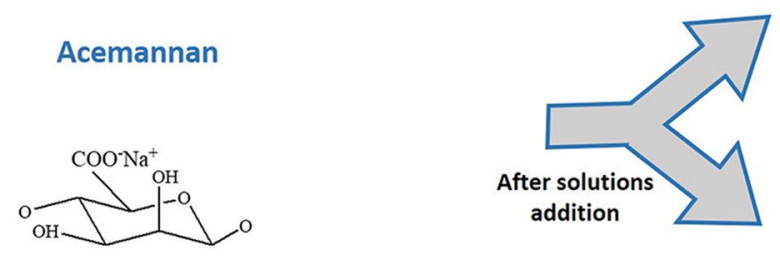

Alginate



ACAL 2

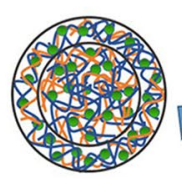

ACAL 1

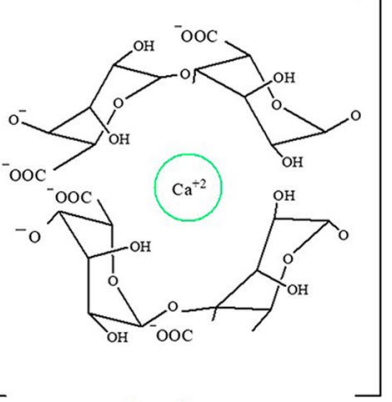

Egg-box



Hydrophilic/hydrophobic

Beads formation

(B)
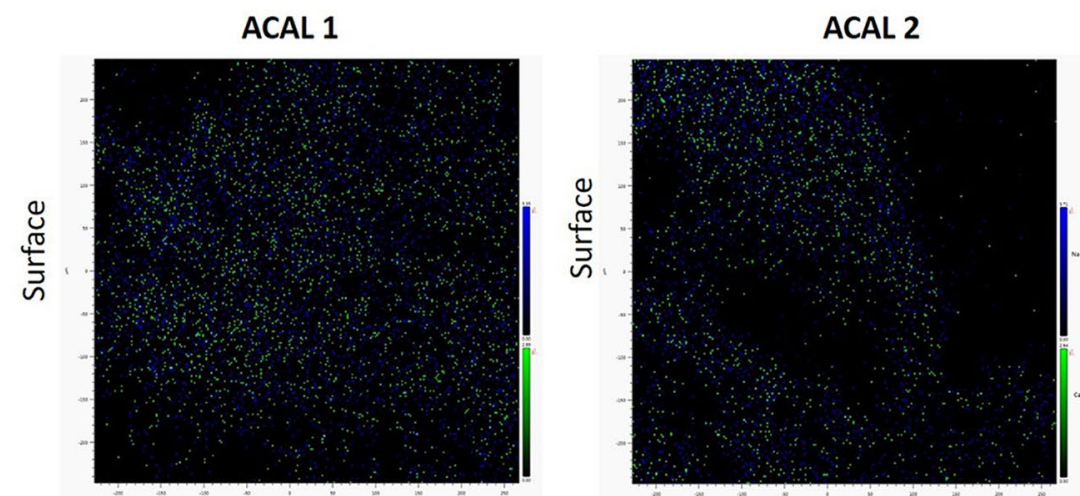

ACE
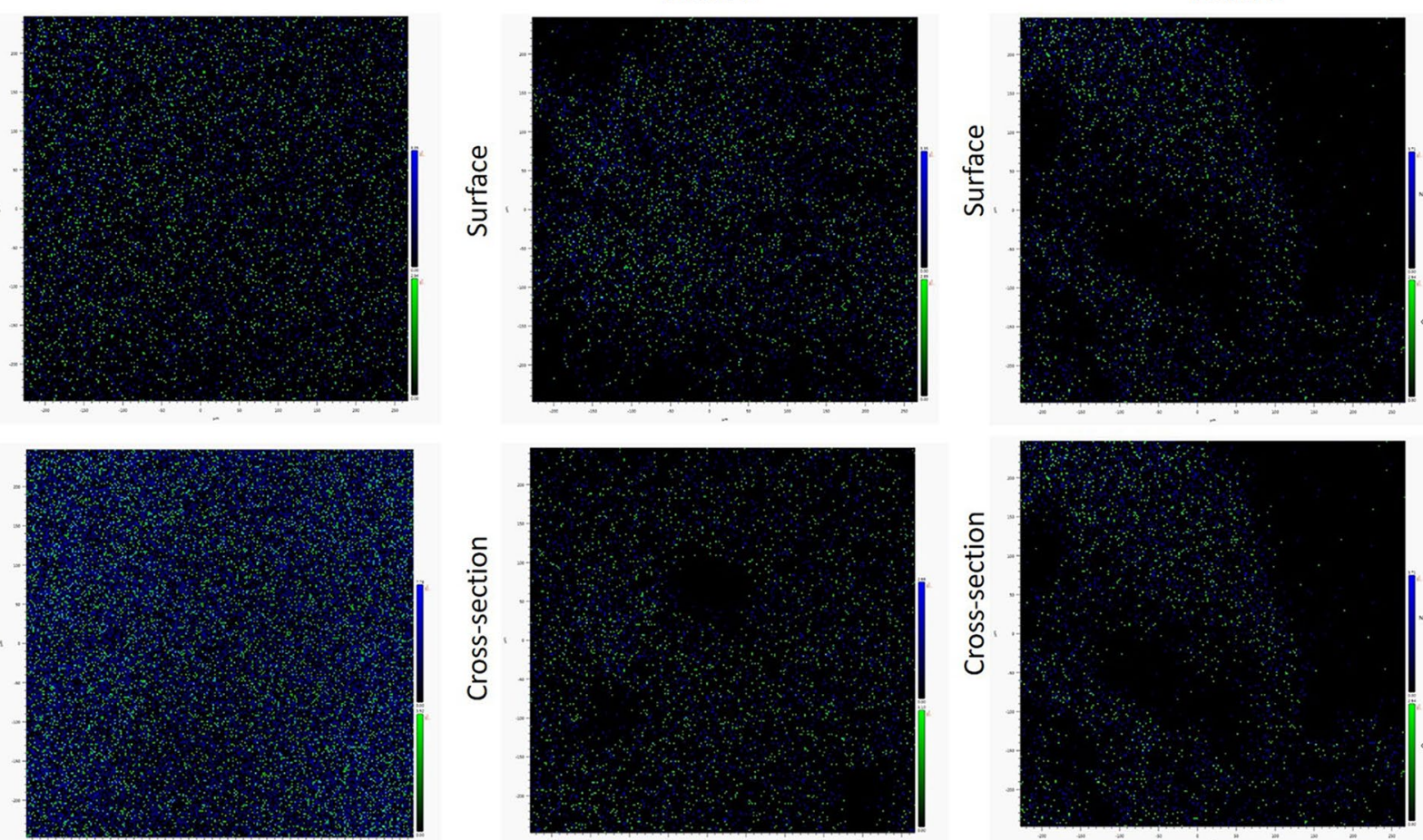

Fig. 6 A Schematic representation of the structures formed by alginate in association with ACE; B XPS imaging of the acemannan, alginate, and their blends 
Surface
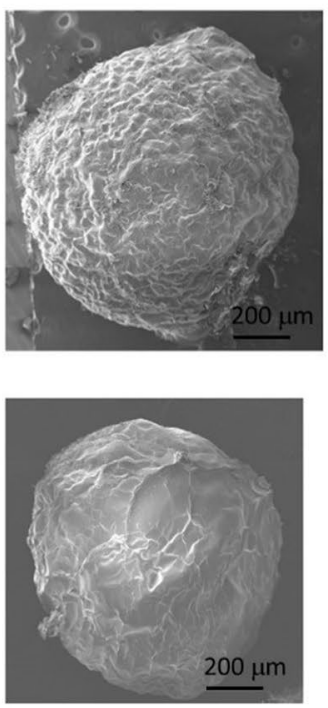

(A)

Cross-section
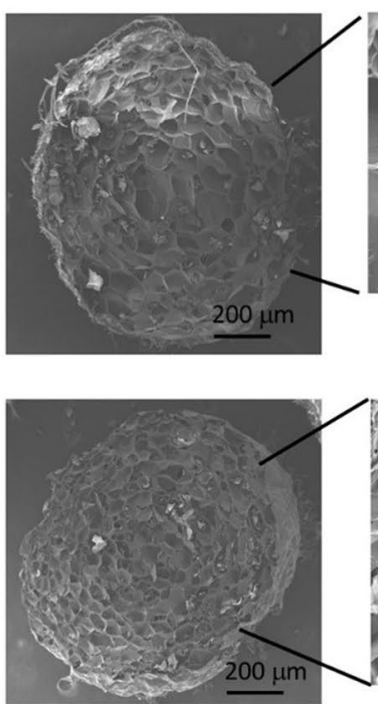
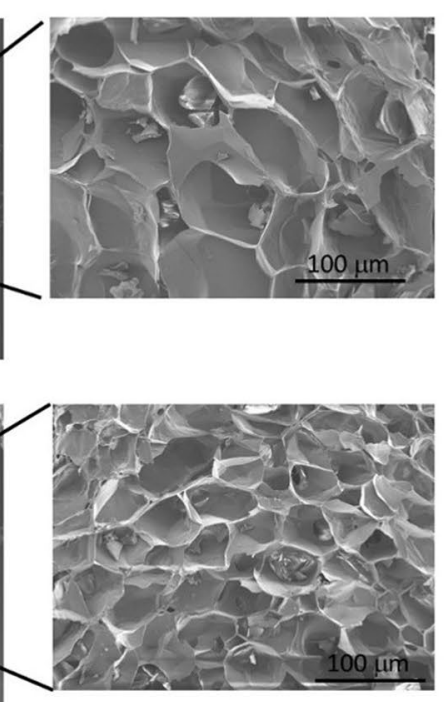

(B)


Fig. 7 A SEM micrographs of the ACAL 1 and ACAL 2 beads; DSC thermograms of $\mathbf{B}$ alginate (ALG), acemannan (ACE) and C ACAL1, ACAL2

\section{Conclusion}

As a proof-of-concept, polymeric beads were successfully produced using a gelling system composed of alginate and acemannan. The characterization of the systems confirmed that the presence of $\mathrm{Ca}^{2+}$ in acemannan composition not only promoted the gel formation but also lead to a reinforcement of their mechanical properties. The developed beads had a spherical shape, micro/ nano- roughness on their surface and displayed a heterogeneous porous formation in their interior. Beyond that, the stronger interaction between alginate and acemannan resulted in beads with higher resistance to thermal degradation than their components. Then, the present approach could improve not only the processability of acemannan but also the production of high added-value structures through a feasible and straightforward platform to design 3D biomaterials that will act at different stages of the tissue regeneration.

Acknowledgements Simone S. Silva and L. C. Rodrigues acknowledge financial support from Portuguese Foundation for Science and Technology-FCT (Grant SFRH/BPD/112140/2015 and SFRH/BPD/93697/2013) and European Union Seventh Framework Programme (FP7/2007-2013) under Grant Agreement No.REGPOT-CT2012-316331-POLARIS. The authors would like to express thanks to Lorand Laboratories (Texas, USA) for the Acemmann (BiAloe ${ }^{\circledast}$ ) supply.

\section{Compliance with ethical standards}

Conflict of interest The authors declare that they have no conflict of interest.

\section{References}

1. Hamman JH (2008) Composition and applications of Aloe vera leaf gel. Molecules 13:1599-1616

2. Balaji A, Vellayappan MV, John AA, Subramanian AP, Jaganathan SK, SelvaKumar M, Mohd Faudzi AA, Supriyanto E, Yusof M (2015) Biomaterials based nano-applications of Aloe vera and its perspective: a review. RSC Adv 5:86199-86213

3. Silva SS, Caridade SG, Mano JF, Reis RL (2013) Effect of crosslinking in chitosan/aloe vera-based membranes for biomedical applications. Carbohydr Polym 98:581-588

4. Silva SS, Popa EG, Gomes ME, Cerqueira M, Marques AP, Caridade SG, Teixeira P, Sousa C, Mano JF, Reis RL (2013) An investigation of the potential application of chitosan/aloe-based membranes for regenerative medicine. Acta Biomater 9:6790-6797

5. Jettanacheawchankit S, Sasithanasate S, Sangvanich P, Banlunara W, Thunyakitpisal P (2009) Acemannan stimulates gingival fibroblast proliferation; expressions of keratinocyte growth factor-1, vascular endothelial growth factor, and type I collagen; and wound healing. J Pharmacol Sci 109:525-531

6. Dusida S, Suparaporn K, Polkit S, Takashi T, Masae K, Pasutha T (2012) Acemannan induces cementoblast proliferation, differentiation, extracellular matrix secretion, and mineral deposition. J Med Plants Res 6:4069-4076

7. Boonyagul S, Banlunara W, Sangvanich $P$, Thunyakitpisal $P$ (2014) Effect of acemannan, an extracted polysaccharide from Aloe vera, on BMSCs proliferation, differentiation, extracellular matrix synthesis, mineralization, and bone formation in a tooth extraction model. Odontology 102:310-317 
8. Escobedo-Lozano AY, Domard A, Velazquez CA, Goycoolea FM, Arguelles-Monal WM (2015) Physical properties and antibacterial activity of chitosan/acemannan mixed systems. Carbohydr Polym 115:707-714

9. Silva SS, Costa SD, Reis RL (2019) Photocrosslinked acemannanbased 3D matrices for in vitro cell culture. J Mater Chem B. https ://doi.org/10.1039/C9TB00593E

10. Alves NM, Picart C, Mano JF (2009) Self assembling and crosslinking of polyelectrolyte multilayer films of chitosan and alginate studied by QCM and IR spectroscopy. Macromol Biosci 9:776-785

11. Lee KY, Mooney DJ (2012) Alginate: properties and biomedical applications. Prog Polym Sci 37:106-126

12. Benselfelt $T$, Engström J, Wågberg $L$ (2018) Supramolecular double networks of cellulose nanofibrils and algal polysaccharides with excellent wet mechanical properties. Green Chem 20:2558-2570

13. Choi EJ, Ha S, Lee J, Premkumar T, Song C (2018) UV-mediated synthesis of pNIPAM-crosslinked double-network alginate hydrogels: enhanced mechanical and shape-memory properties by metal ions and temperature. Polymer 149:206-212

14. Segale L, Giovannelli L, Mannina P, Pattarino F (2016) Calcium alginate and calcium alginate-chitosan beads containing celecoxib solubilized in a self-emulsifying phase. Scientifica 2016:8

15. Kilicarslan M, Ilhan M, Inal O, Orhan K (2018) Preparation and evaluation of clindamycin phosphate loaded chitosan/alginate polyelectrolyte complex film as mucoadhesive drug delivery system for periodontal therapy. Eur J Pharm Sci 123:441-451

16. Baysal K, Aroguz AZ, Adiguzel Z, Baysal BM (2013) Chitosan/ alginate crosslinked hydrogels: preparation, characterization and application for cell growth purposes. Int J Biol Macromol 59:342-348

17. Sun J, Tan H (2013) Alginate-based biomaterials for regenerative medicine applications. Materials 6:1285-1309
18. Shon SO, Ji BC, Han YA, Park D-J, Kim IS, Choi JH (2007) Viscoelastic sol-gel state of the chitosan and alginate solution mixture. J Appl Polym Sci 104:1408-1414

19. Castle JE (1984) Practical surface analysis by Auger and X-ray photoelectron spectroscopy. D. Briggs and M. P. Seah (Editors). John Wiley and Sons Ltd, Chichester, 1983, 533 pp., £44.50. Surf Interface Anal 6(6):302. https://doi.org/10.1002/sia.740060611

20. Khong TT, Aarstad OA, Skjåk-Bræk G, Draget KI, Vårum KM (2013) Gelling concept combining chitosan and alginate-proof of principle. Biomacromolecules 14:2765-2771

21. Voo W-P, Ooi C-W, Islam A, Tey B-T, Chan E-S (2016) Calcium alginate hydrogel beads with high stiffness and extended dissolution behaviour. Eur Polym J 75:343-353

22. Caridade SG, Monge C, Gilde F, Boudou T, Mano JF, Picart C (2013) Free-standing polyelectrolyte membranes made of chitosan and alginate. Biomacromolecules 14:1653-1660

23. Bartnikowski M, Wellard R, Woodruff M, Klein T (2015) Tailoring hydrogel viscoelasticity with physical and chemical crosslinking. Polymers 7:2650-2669

24. Lawrie G, Keen I, Drew B, Chandler-Temple A, Rintoul L, Fredericks P, Grøndahl L (2007) Interactions between alginate and chitosan biopolymers characterized using FTIR and XPS. Biomacromolecules 8:2533-2541

25. Quezada MP, Salinas C, Gotteland M, Cardemil L (2017) Acemannan and fructans from Aloe vera (Aloe barbadensis Miller) plants as novel prebiotics. J Agric Food Chem 65:10029-10039

26. Pereira R, Mendes A, Bártolo P (2013) Alginate/Aloe vera hydrogel films for biomedical applications. Procedia CIRP 5:210-215

Publisher's Note Springer Nature remains neutral with regard to jurisdictional claims in published maps and institutional affiliations. 